hep-ph/yymmnnn

\title{
Photonic SUSY Two-Loop Corrections to the Muon Magnetic Moment
}

\author{
P. von Weitershausen ${ }^{a}$, M. Schäfer ${ }^{b}$, H. Stöckinger-Kim ${ }^{c}$, \\ D. STÖCKINGER ${ }^{c}$ \\ ${ }^{a}$ Department of Physics and Astronomy, University of Glasgow, Glasgow, UK \\ ${ }^{b}$ Theoretisch-Physikalisches Institut, Universität Jena, Jena, Germany \\ 'Institut für Kern- und Teilchenphysik, TU Dresden, Dresden, Germany
}

\begin{abstract}
Photonic SUSY two-loop corrections to the muon magnetic moment are contributions from diagrams where an additional photon loop is attached to a SUSY one-loop diagram. These photonic corrections are evaluated exactly, extending a leading-log calculation by Degrassi and Giudice. Compact analytical expressions are provided and the numerical behaviour is discussed. The photonic corrections reduce the SUSY one-loop result by $7 \ldots 9 \%$. The new terms are typically around ten times smaller than the leading logarithms, but they can be larger and have either sign in cases with large SUSY mass splittings. We also provide details on renormalization and regularization and on how to incorporate the photonic corrections into a full SUSY two-loop calculation.
\end{abstract}

PACS numbers: 12.20.Ds, 12.60.Jv, 13.40.Em, 14.60.Ef 


\section{Introduction}

The muon magnetic dipole moment belongs to the most precisely known observables in particle physics. The anomalous magnetic moment $a_{\mu}=(g-2)_{\mu} / 2$ has been determined by an impressive series of measurements at BNL to [1]

$$
a_{\mu}^{\exp }=(11659208.9 \pm 6.3) \times 10^{-10} .
$$

At this level of precision, $a_{\mu}$ is sensitive to all interactions of the Standard Model $(\mathrm{SM})$ as well as to hypothetical new particles at the electroweak scale.

In recent years, the precision of the Standard Model (SM) theory evaluation has reached a similar level. The hadronic vacuum polarization contributions can be related to the cross section for $e^{+} e^{-} \rightarrow$ hadrons in a theoretically clean way, and crucial recent progress has been achieved on the experimental determination of this cross section by the SND, CMD-2, KLOE, and Babar experiments [3 6]. The quality of the current $e^{+} e^{-} \rightarrow$ hadrons data is such that all recent theory evaluations of the corresponding contributions to $a_{\mu}$ agree fairly well, see e.g. [7. 12 . 2

The hadronic light-by-light contributions have been scrutinized by many groups in the past 15 years, and while an ever better understanding has been achieved, the central value of the result has remained relatively stable. Recently, three groups have joined forces and published a common value, $a_{\mu}^{\text {lbl }}=$ $10.5(2.6) \times 10^{-10}$ [17], where the errors have been enlarged in order to cover the results obtained from the different approaches. Compatible results have been obtained even more recently in Refs. [10, 18].

As a result of the recent progress, the SM theory prediction for $a_{\mu}$ now has an even smaller error than $a_{\mu}^{\exp }$, calling for a new experiment. For reference we use the value from [12],

$$
a_{\mu}^{\mathrm{SM}}=(11659183.4 \pm 4.9) \times 10^{-10} .
$$

The deviation from the experimental value is

$$
\Delta a_{\mu}(\exp -\mathrm{SM})=(25.5 \pm 8.0) \times 10^{-10} .
$$

All the other mentioned recent $e^{+} e^{-}$-based evaluations lead to similar deviations in the range $(25.5 \ldots 31.6) \times 10^{-10}$ with combined errors in the range $(7.9 \ldots 9.0) \times$ $10^{-10}$.

\footnotetext{
${ }^{1}$ The change in the number compared to Ref. [1] is due to a new PDG value for the magnetic moment ratio of the muon to proton [2].

${ }^{2}$ In principle, part of the $e^{+} e^{-} \rightarrow$ hadrons cross section could be obtained in an alternative way from hadronic $\tau$ decays [13]. However, the required isospin breaking effects are difficult to quantify at the currently needed level of precision [10, 14, 16. Hence, in order to obtain the most reliable results, most analyses do not use $\tau$ decays. Nevertheless, the most recent analysis [1] employing an improved understanding of isospin breaking effects shows a marginal consistency between the $e^{+} e^{-}$-based and the $\tau$-decay based evaluations of $a_{\mu}$.
} 
This $3-4 \sigma$ deviation constitutes a tantalizing hint for physics beyond the SM. Although the deviation is almost twice as large as the SM weak contributions $a_{\mu}^{\text {weak }}$ and contributions from hypothetical heavy particles with mass $M$ are typically suppressed $\propto\left(M_{W} / M\right)^{2} a_{\mu}^{\text {weak }}$, there is a variety of models that could explain it [19. Supersymmetry (SUSY) is a particularly promising example (for a review see [20]), owing to an enhancement by $\tan \beta$, the ratio of the two Higgs vacuum expectation values.

Even more importantly, the precision of the deviation (3) implies significant constraints on the parameters of many new physics models. In supersymmetry, $a_{\mu}$ permits to derive mass limits can which cannot be obtained from other observables [21; $a_{\mu}$ is also one of the most important quantities in recent global analyses of the parameter space of various supersymmetric models [22 25]. Even in the LHC era, $a_{\mu}$ will remain a highly useful complementary observable. It will provide a benchmark for models, help selecting between different SUSY scenarios, eliminate ambiguities, and it will improve parameter determinations. For instance, combining LHC data with $a_{\mu}$ will significantly reduce the $\tan \beta$ uncertainty [26 28].

Given the current situation and the usefulness of $a_{\mu}$, every effort should be made in order to improve the experimental and theoretical precision of $a_{\mu}$. In the near future, the precision of $\Delta a_{\mu}(\exp -\mathrm{SM})$ will indeed further increase. New analyses of $e^{+} e^{-} \rightarrow$ hadrons data are in the pipeline, and a new CMD-3 experiment in Novosibirsk is planned. All of these will directly feed into a more precise evaluation of the hadronic vacuum polarization contributions. Furthermore, a new, improved measurement of $a_{\mu}$ itself could be carried out at Fermilab [29] or possibly at J-PARC [30]. Building on the BNL experiment, whose final uncertainty is still statistics dominated, the Fermilab measurement could reach a reduction of the uncertainty down to $1.6 \times 10^{-10}$.

With these expected improvements, the theory error from contributions from physics beyond the SM, in particular from supersymmetry, becomes more prominent. The current theory error of the SUSY contributions has been estimated to $3 \times 10^{-10}$ [20], which is larger than the precision goal of the Fermilab $a_{\mu}$ measurement.

The one-loop contributions from supersymmetric particles have been computed a long time ago [31,32], and the SUSY two-loop corrections to SM oneloop diagrams (e.g. SM-diagrams with insertions of closed chargino/neutralino or stop/sbottom loops) are completely known [33 35]. However, the two-loop corrections to SUSY one-loop diagrams are not known, only two dominant parts have been identified: large QED-logarithms [36] and $(\tan \beta)^{2}$-enhanced corrections [37]. A subclass of the remaining diagrams has been considered in Ref. [38]. The unknown two-loop contributions lead to the theory error mentioned above.

In the present paper we consider the photonic two-loop contributions, defined as contributions from diagrams where a photon loop is attached to a SUSY oneloop diagram. In Ref. [36], Degrassi and Giudice showed that these contributions 
lead to large QED-logarithms of the form $\log \left(M / m_{\mu}\right)$, where $M$ is the mass scale of the new particles. They evaluated the logarithms using elegant effective field theory and renormalization group techniques. These logarithms reduce the oneloop contributions by $7 \ldots 9 \%$ for $M$ between 100 and $1000 \mathrm{GeV}$. It is clear that there is an ambiguity in the choice of $M$, in particular in the case of a rather split spectrum. In the present paper we evaluate the photonic two-loop corrections exactly. In this way, we resolve the ambiguity, and we obtain all logarithms of ratios of different heavy masses and the associated non-logarithmic terms. Our results are derived in a generic model which covers the case of the minimal supersymmetric SM (MSSM) but also of a wider class of models. We take into account issues such as the choice of dimensional regularization versus dimensional reduction, and we set up the calculation in such a way that our results will be useful building blocks for a full two-loop computation of $a_{\mu}^{\text {SUSY }}$.

The outline of the paper is as follows. In section 2 we describe the setup of our calculation and provide the necessary one-loop contributions up to the order $\epsilon$ on the regularized level. In section 3 we list the contributing two-loop diagrams, classify them according to their ultraviolet (UV) and infrared (IR) divergences, and explain our method to compute them. Section 4 is devoted to renormalization and the analysis of the counterterm diagrams. We provide details on the cancellation of UV and IR divergences and the regularizationscheme dependence. In section 5 we provide our results as a compact analytical formula, and discuss several numerical examples. In section 6 we discuss how our results can be used as building blocks in a full MSSM two-loop calculation. Section 7 contains the conclusions.

\section{Generic model and one-loop contributions}

The genuine SUSY one-loop contributions to $a_{\mu}$ in SUSY extensions of the SM are given by the two kinds of diagrams in Fig. 11. The loops involve either a chargino $\chi^{ \pm}$and a sneutrino $\tilde{\nu}_{\mu}$ or a neutralino $\chi^{0}$ and a smuon $\tilde{\mu}$. At the two-loop level it is useful to classify the SUSY contributions into those from diagrams where the $\mu$-lepton number is only carried by muon or muon-neutrino, and diagrams where the $\mu$-lepton number is carried also by $\tilde{\mu}$ and/or $\tilde{\nu}_{\mu}$. The second class can be interpreted as the two-loop corrections to the SUSY one-loop diagrams in Fig. 1.

In the present paper we aim for the computation of the photonic contributions of this second class, i.e. of two-loop diagrams obtained from attaching a photon loop in all possible ways to the SUSY one-loop diagrams. The relevant Lagrangian 

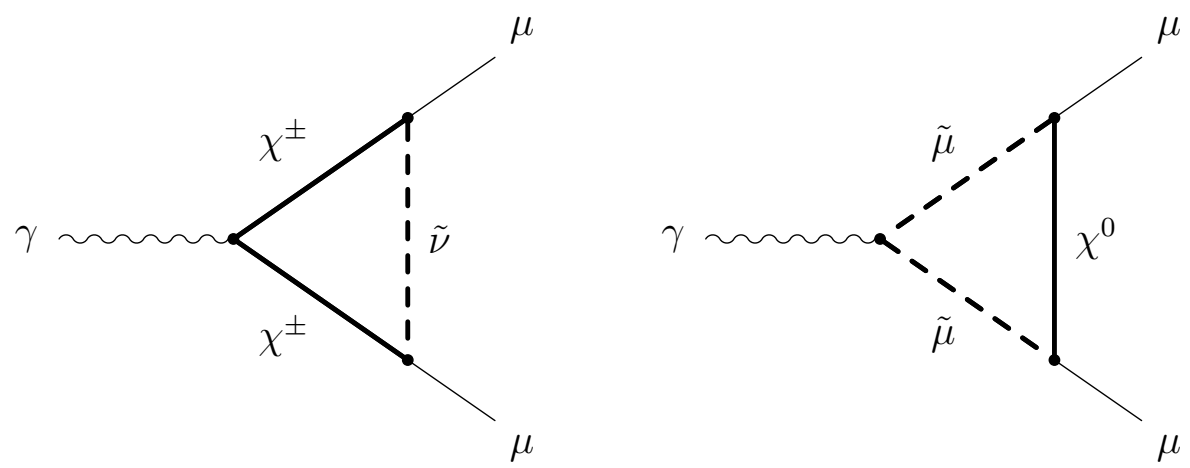

Figure 1: The two one-loop diagrams involving SUSY particles. In this and all following figures, thin lines denote the photon and muon, thick lines denote the chargino/neutralino, thick dashed lines the sneutrino/smuon.

is given by

$$
\begin{aligned}
\mathcal{L}_{\mathrm{QED}+c, n} & =\mathcal{L}_{\mathrm{QED}}+\mathcal{L}_{\text {mat }}+\mathcal{L}_{\text {int }}, \\
\mathcal{L}_{\text {mat }} & =\overline{\chi^{-}}\left(i \not D-m_{\chi^{ \pm}}\right) \chi^{-}+\left|D^{\mu} \tilde{\mu}\right|^{2}-m_{\tilde{\mu}}^{2}|\tilde{\mu}|^{2} \\
& +\frac{1}{2} \overline{\chi^{0}}\left(i \not \supset-m_{\chi^{0}}\right) \chi^{0}+\left|\partial^{\mu} \tilde{\nu}\right|^{2}-m_{\tilde{\nu}}^{2}|\tilde{\nu}|^{2}, \\
\mathcal{L}_{\text {int }} & =\tilde{\nu}^{\dagger} \overline{\chi^{-}}\left(c_{L}^{*} P_{L}+c_{R} P_{R}\right) \mu+\tilde{\mu}^{\dagger} \overline{\chi^{0}}\left(n_{L}^{*} P_{L}-n_{R} P_{R}\right) \mu+\text { h.c.. }
\end{aligned}
$$

Here $\mathcal{L}_{\text {QED }}$ is the QED Lagrangian for photon and muon $\mu ; \chi^{-}$and $\chi^{0}$ are the chargino and neutralino spinor fields, $D^{\mu}$ is the covariant derivative in QED and $P_{L, R}=\frac{1}{2}\left(1 \mp \gamma^{5}\right)$. This Lagrangian contains only photon and muon plus one generic chargino, neutralino, sneutrino, and smuon. For the following, it will be useful to adopt the viewpoint that $\mathcal{L}_{\mathrm{QED}+c, n}$ describes a simple generic model which extends QED by two charged and two uncharged particles. The couplings of the SUSY particles to the muon are given by free parameters $c^{L, R}$ and $n^{L, R}$, all other terms in the Lagrangian are determined by QED gauge invariance. The conventions of the couplings $c, n$ are chosen in the same way as in Ref. [39]. In terms of this generic model, the one-loop contributions in Fig. 1 and the photonic two-loop corrections (plus renormalization) are defined as the contributions of $\mathcal{O}\left(c^{2}, n^{2}\right)$ and $\mathcal{O}\left(\alpha c^{2}, \alpha n^{2}\right)$, respectively, with the fine structure constant $\alpha=$ $e^{2} / 4 \pi$.

By specializing the couplings, the generic model can describe photonic corrections in underlying models such as the MSSM, but also in non-minimal SUSY models, and even in non-supersymmetric models, if they happen to contribute to $a_{\mu}$ by diagrams of the form in Fig. 1. For instance, in the MSSM, the couplings 
$c^{L, R}$ and $n^{L, R}$ have the following values:

$$
\begin{aligned}
c_{k}^{L} & =-g_{2} V_{k 1}, \\
c_{k}^{R} & =y_{\mu} U_{k 2}, \\
n_{i m}^{L} & =\frac{1}{\sqrt{2}}\left(g_{1} N_{i 1}+g_{2} N_{i 2}\right) U_{m 1}^{\tilde{\mu}}{ }^{*}-y_{\mu} N_{i 3} U_{m 2}^{\tilde{\mu}}{ }^{*}, \\
n_{i m}^{R} & =\sqrt{2} g_{1} N_{i 1} U_{m 2}^{\tilde{\mu}}+y_{\mu} N_{i 3} U_{m 1}^{\tilde{\mu}},
\end{aligned}
$$

where the chargino, neutralino and smuon indices can take the values $k \in\{1,2\}$, $i \in\{1, \ldots, 4\}, m \in\{1,2\}$, and where the notation of Ref. [20] has been used for gauge and Yukawa couplings and mixing matrices.

As a first step we present the result of the one-loop diagrams of Fig. 1. These diagrams will be needed later as counterterm diagrams, multiplied with $1 / \epsilon$ divergences from renormalization constants, where $D=4-2 \epsilon$ in dimensional regularization. Hence, we do not only need the finite part of the result but also the $\mathcal{O}(\epsilon)$-part.

This $\mathcal{O}(\epsilon)$-part can in principle depend on the regularization scheme. We consider two schemes: dimensional regularization (DREG) and dimensional reduction (DRED). As discussed e.g. in [40], it is crucial to define the treatment of external vector bosons in dimensional schemes. Our definitions are as follows: In DREG, the vertex function $\Gamma_{\mu \bar{\mu} A^{\rho}}$ is computed with a $D$-dimensional photon, then the $D$-dimensional projector to extract $a_{\mu}$ defined in [34,41] is applied. In DRED, the vertex function $\Gamma_{\mu \bar{\mu} A^{\rho}}$ is computed with a (quasi-)4-dimensional photon, then the photon is projected onto the $D$-dimensional subspace, $\hat{g}_{\rho}^{\sigma} \Gamma_{\mu \bar{\mu} A^{\sigma}}$ in the notation of [40], and finally the same $D$-dimensional $a_{\mu}$-projector is applied 3

With these definitions, it is clear that the regularized results for the diagrams in Fig. 1 are equal in DREG and DRED, even at $\mathcal{O}(\epsilon)$. The results read

$$
\begin{aligned}
& a_{\mu}^{\chi^{ \pm}}=\frac{1}{16 \pi^{2}} \frac{m_{\mu}^{2}}{m_{\tilde{\nu}}^{2}}\left\{\frac{1}{12} \mathcal{A}^{C} \mathcal{F}_{1}^{C}(x)+\frac{2 m_{\chi^{ \pm}}}{3} \mathcal{B}^{C} \mathcal{F}_{2}^{C}(x)\right\} \\
& a_{\mu}^{\chi^{0}}=\frac{1}{16 \pi^{2}} \frac{m_{\mu}^{2}}{m_{\tilde{\mu}}^{2}}\left\{-\frac{1}{12} \mathcal{A}^{N} \mathcal{F}_{1}^{N}(x)+\frac{m_{\chi^{0}}}{3} \mathcal{B}^{N} \mathcal{F}_{2}^{N}(x)\right\}
\end{aligned}
$$

with the kinematic variables $x=m_{\chi^{ \pm}}^{2} / m_{\tilde{\nu}_{\mu}}^{2}$, or $x=m_{\chi^{0}}^{2} / m_{\tilde{\mu}}^{2}$, respectively, and the coupling combinations

$$
\begin{array}{ll}
\mathcal{A}^{C}=\left|c^{L}\right|^{2}+\left|c^{R}\right|^{2}, & \mathcal{B}^{C}=\frac{\operatorname{Re}\left[c^{L} c^{R}\right]}{m_{\mu}}, \\
\mathcal{A}^{N}=\left|n^{L}\right|^{2}+\left|n^{R}\right|^{2}, & \mathcal{B}^{N}=\frac{\operatorname{Re}\left[n^{L} n^{R}\right]}{m_{\mu}} .
\end{array}
$$

\footnotetext{
${ }^{3}$ Note that in DRED, for a quasi-4-dimensional photon, the covariant decomposition of $\Gamma_{\mu \bar{\mu} A^{\sigma}}$ has more terms than in DREG, and that different prescriptions to project out $a_{\mu}$ can lead to different results at $\mathcal{O}(\epsilon)$.
} 
The $\epsilon$-dependent loop functions are decomposed as

$$
\begin{aligned}
& \mathcal{F}_{i}^{C}(x)=F_{i}^{C}(x)\left[1-\epsilon \mathrm{L}\left(m_{\tilde{\nu}_{\mu}}^{2}\right)\right]+\epsilon F_{i \epsilon}^{C}(x), \\
& \mathcal{F}_{i}^{N}(x)=F_{i}^{N}(x)\left[1-\epsilon \mathrm{L}\left(m_{\tilde{\mu}}^{2}\right)\right]+\epsilon F_{i \epsilon}^{N}(x),
\end{aligned}
$$

where we have used the abbreviation

$$
\mathrm{L}\left(m^{2}\right)=\log \frac{m^{2}}{\mu_{\text {DREG }}^{2}}
$$

with the dimensional-regularization scale $\mu_{\mathrm{DREG}}$, and the well-known functions

$$
\begin{aligned}
F_{1}^{C}(x) & =\frac{2}{(1-x)^{4}}\left[2+3 x-6 x^{2}+x^{3}+6 x \log x\right], \\
F_{2}^{C}(x) & =\frac{3}{2(1-x)^{3}}\left[-3+4 x-x^{2}-2 \log x\right], \\
F_{1}^{N}(x) & =\frac{2}{(1-x)^{4}}\left[1-6 x+3 x^{2}+2 x^{3}-6 x^{2} \log x\right], \\
F_{2}^{N}(x) & =\frac{3}{(1-x)^{3}}\left[1-x^{2}+2 x \log x\right],
\end{aligned}
$$

normalized such that $F_{i}^{j}(1)=1$. The functions for the $\epsilon$-dependent parts are defined as

$$
\begin{aligned}
& F_{1 \epsilon}^{C}(x)=F_{1}^{C}(x)\left(\frac{-x^{3}+6 x^{2}+15 x+2-6 x \log x}{12 x}\right)+\frac{x^{2}-8 x-4}{6 x}, \\
& F_{2 \epsilon}^{C}(x)=F_{2}^{C}(x)\left(\frac{-2 x^{2}+8 x+6-4 \log x}{8}\right)+\frac{3 x-15}{8} \\
& F_{1 \epsilon}^{N}(x)=F_{1}^{N}(x)\left(\frac{2 x^{3}+15 x^{2}+6 x-1-6 x^{2} \log x}{12 x^{2}}\right)+\frac{1-8 x-4 x^{2}}{6 x^{2}}, \\
& F_{2 \epsilon}^{N}(x)=F_{2}^{N}(x)\left(\frac{x^{2}+4 x+1-2 x \log x}{4 x}\right)-\frac{3 x+3}{4 x}
\end{aligned}
$$

and are normalized to $F_{i \epsilon}^{j}(1)=0$. The $\mathcal{O}\left(\epsilon^{0}\right)$-part of this result has been presented in a form similar to the one in [39].

Our definition of the coupling combinations $\mathcal{A}, \mathcal{B}$ reflects an important physical property. The muon anomalous magnetic moment, like the muon mass, corresponds to a chirality-flipping operator, and all contributions must be proportional to a chiral-symmetry violating parameter. In the SM, the MSSM and many other models, chiral symmetry in the muon sector is broken only by the muon mass and its Yukawa coupling. In the remainder of the article we assume nothing about the model underlying the Lagrangian (4) except that it has this same property. 
With this assumption, all contributions to $a_{\mu}$ are of the order $m_{\mu}^{2} / M_{\text {SUSY }}^{2}$ in the muon mass, like in the MSSM [19,20, 32]. Furthermore, the combinations $\mathcal{A}^{C, N}$ and $\mathcal{B}^{C, N}$ must have the behaviour

$$
\mathcal{A}^{C, N}, \mathcal{B}^{C, N}=\text { const. }+\mathcal{O}\left(m_{\mu}^{2}\right)
$$

in the muon mass. In other words, either $c^{L}$ or $c^{R}$ must be proportional to the muon mass, and similar for $n^{L}, n^{R}$. It is noteworthy that an equivalent assumption has been made implicitly in Ref. [36] in the computation of the 2-loop QED-logarithms in new physics models using effective field theory techniques. In the matching between the full and the effective theory Ref. [36] assumed that one can pull a factor $m_{\mu}^{2}$ out of the new physics contributions to $a_{\mu}$. If that were not the case, additional large QED-logarithms would appear in the matching.

For the case of the MSSM, Eq. (77) shows that the couplings indeed have the assumed behaviour, for all values of the chargino, neutralino and smuon indices 4 . For phenomenology it is important that $\mathcal{B}^{C, N}$ are enhanced by a factor $\tan \beta$. In a large part of the MSSM parameter space, the $\mathcal{B}^{C}$-term in $a_{\mu}^{\chi^{ \pm}}$constitutes the dominant SUSY contribution to $a_{\mu}$.

\section{Two-loop contributions}

We are interested in the photonic two-loop corrections to Fig. 1, i.e. the two-loop contributions of $\mathcal{O}\left(\alpha c^{2}, \alpha n^{2}\right)$ in our generic model. We denote the corresponding genuine two-loop diagrams by $a_{\mu}^{2 \mathrm{~L} \operatorname{diag}(\gamma)}$. They are shown in Figs. 2, 3, divided into diagrams with chargino/sneutrino or neutralino/smuon exchange. All diagrams are overall UV-convergent, but a useful classification according to divergent subdiagrams is possible.

- The diagrams in Figs. 2a and 3a contain a UV-divergent SUSY subdiagram, inserted into a QED diagram.

- The diagrams in Figs. 2b and 3b contain a UV-divergent QED subdiagram, inserted into a SUSY diagram. There are more neutralino/smuon diagrams because of the presence of vertices involving two photons.

- The diagrams in Figs. 2cc and 3rc contain no UV-divergent subdiagrams and are finite.

There are also IR divergences in the third diagrams in Figs. 2a and 3a, which are the only diagrams where both ends of the photon propagator are attached to the external muons and with a subdiagram that contributes to $a_{\mu}$.

\footnotetext{
${ }^{4}$ In order to see this, note that the muon Yukawa coupling $y_{\mu}$ and, for each $m$, one of the mixing matrix elements $U_{m 1}^{\tilde{\mu}}$ or $U^{\tilde{\mu}}{ }_{m 2}$ are proportional to $m_{\mu}$.
} 
(a)
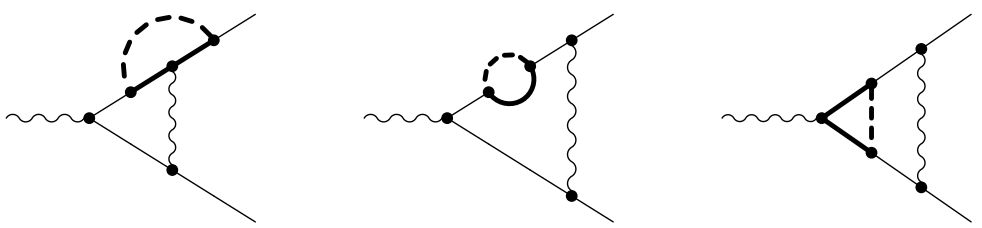

(b)
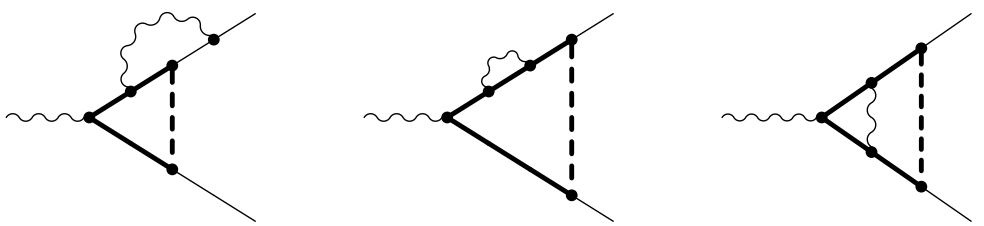

(c)

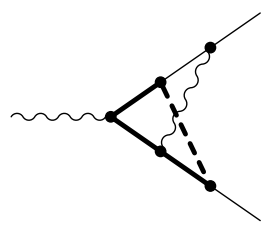

Figure 2: Photonic two-loop corrections to chargino one-loop diagrams. In this and all following figures, it is understood that many graphs (here all graphs except the 3rd and 6th) appear twice, with the SUSY loop either on the upper or on the lower muon line. These graphs are displayed only once.

(a)
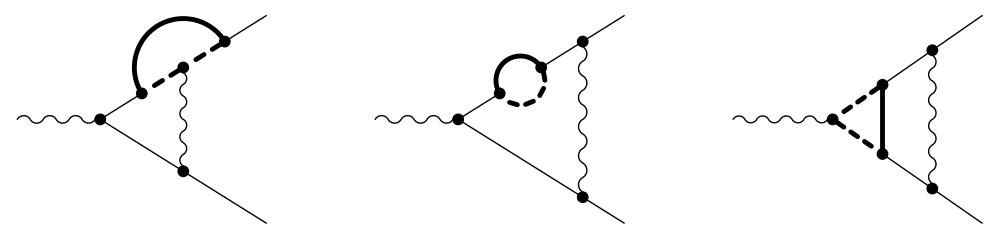

(b)
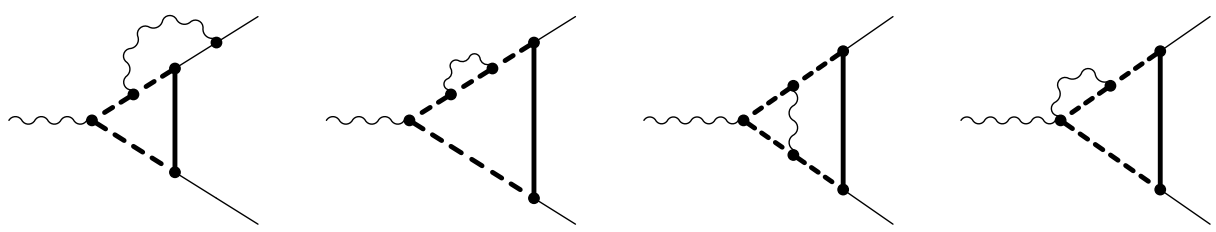

(c)
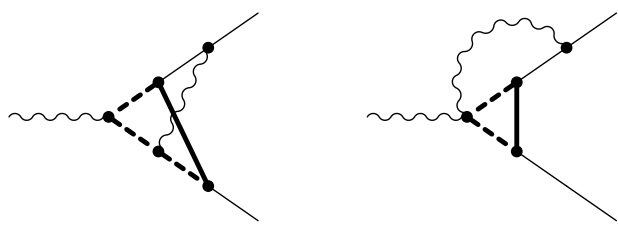

Figure 3: Photonic two-loop corrections to neutralino one-loop diagrams. 

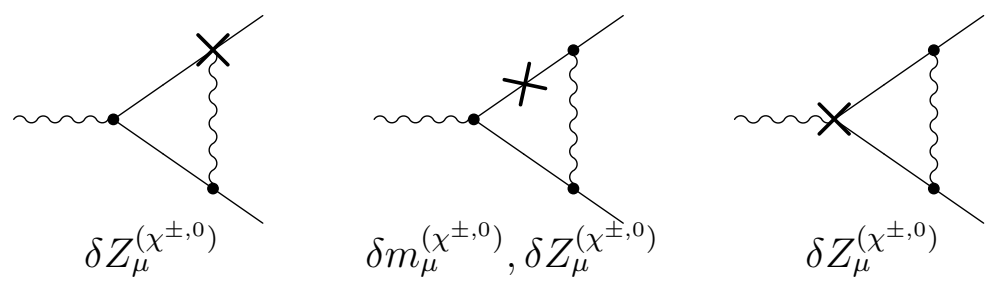

Figure 4: The QED counterterm diagrams and relevant renormalization constants. In the first and third diagram also $\delta e^{\left(\chi^{ \pm, 0}\right)}, \delta Z_{A}^{\left(\chi^{ \pm, 0}\right)}$ would appear, but these vanish.

We have evaluated the diagrams using DREG as a regulator for UV and IR divergences. In the next section we will discuss how the UV and IR divergences of the different classes of diagrams are cancelled by renormalization, and we will discuss the transition to DRED. Technically, we have employed computer algebra programs based on the packages FeynArts [42] (using both a custom model file and the standard MSSM model file [43]) and Two Calc [44] (with a custom routine for the integral reduction).

We have applied a large mass expansion [45] to all diagrams, where the muon mass is treated as small and all other masses as large. This results in a separation of heavy and light scales, and after reduction to master integrals only two kinds of loop integrals can appear:

- products of a light one-loop integral (depending only on $m_{\mu}$ ) and a heavy one-loop integral (independent of $m_{\mu}$ ): these are the integrals in which the large $\log m_{\mu}$-factors considered in [36] but also other terms are generated.

- heavy two-loop integrals (independent of $m_{\mu}$, at most multiplied with polynomials in $m_{\mu}$ ): no large logarithms are generated here; these contributions are new. The heavy two-loop integrals have no external momentum and can depend only on two different mass scales.

The structure of these integrals makes clear that $\log m_{\mu}$-factors appear in all diagrams in which the internal photon couples to at least one muon. Furthermore, the diagrams of Figs. 2 a and 3 a contain terms of order $\left(m_{\mu} / M_{\mathrm{SUSY}}\right)^{0}$, while all other diagrams are suppressed by the small ratio $\left(m_{\mu} / M_{\mathrm{SUSY}}\right)^{2}$. The final result of all diagrams can be expressed as a rational function of the particle masses, and logarithms and dilogarithms. Below we will present the result obtained after renormalization.

\section{Renormalization and counterterms}

Apart from the actual two-loop diagrams, two kinds of counterterm diagrams can contribute at $\mathcal{O}\left(\alpha n^{2}, \alpha c^{2}\right)$, and we denote the corresponding contributions as 

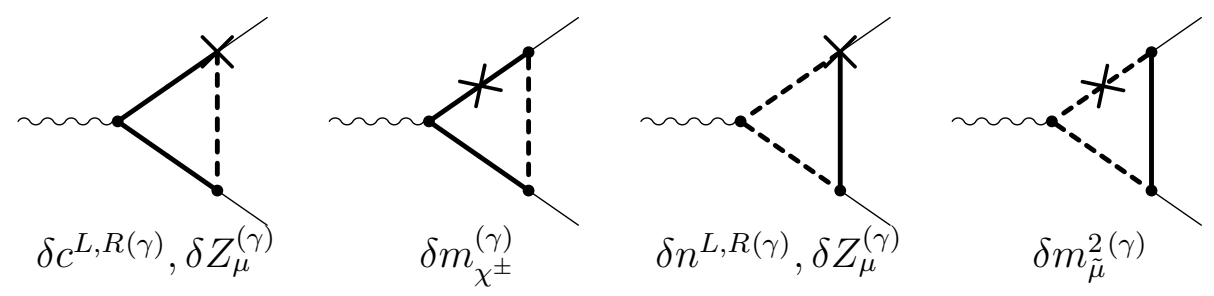

Figure 5: The non-vanishing SUSY counterterm diagrams and relevant renormalization constants.

$a_{\mu}^{\mathrm{ct}, \mathrm{QED}}+a_{\mu}^{\mathrm{ct}, \mathrm{SUSY}}$. The QED counterterm diagrams in Fig. 4, $a_{\mu}^{\mathrm{ct}, \mathrm{QED}}$, arise from renormalization of the QED quantities in the model Lagrangian and involve the renormalization constants

$$
\delta m_{\mu}^{\left(\chi^{ \pm, 0}\right)}, \delta Z_{\mu}^{\left(\chi^{ \pm, 0}\right)}, \delta e^{\left(\chi^{ \pm, 0}\right)}, \delta Z_{A}^{\left(\chi^{ \pm, 0}\right)}
$$

for muon mass and field renormalization, charge and photon field renormalization. The superscripts indicate that these QED renormalization constants need to be evaluated at $\mathcal{O}\left(c^{2}, n^{2}\right)$, from SUSY one-loop diagrams involving a chargino-sneutrino or neutralino-smuon loop. This implies in particular $\delta Z_{A}^{\left(\chi^{ \pm, 0}\right)}=\delta e^{\left(\chi^{ \pm, 0}\right)}=0$. The values of the renormalization constants, and the physical meaning of the renormalized parameters, are fixed by the choice of a renormalization scheme. All QED quantities, in particular $\delta m_{\mu}^{\left(\chi^{ \pm, 0}\right)}$ and $\delta Z_{\mu}^{\left(\chi^{ \pm, 0}\right)}$ must be defined in the on-shell scheme in order to guarantee the correct relation between the 3-point function and $a_{\mu}$.

The SUSY counterterm diagrams in Fig. 15, $a_{\mu}^{\mathrm{ct}, \mathrm{SUSY}}$, arise from renormalization of the SUSY quantities and involve the renormalization constants

$$
\delta m_{\chi^{ \pm}}^{(\gamma)}, \delta m_{\tilde{\mu}}^{2(\gamma)}, \delta c^{L, R(\gamma)}, \delta n^{L, R(\gamma)} ; \quad \delta Z_{\mu}^{(\gamma)}
$$

for SUSY mass and coupling renormalization and muon field renormalization. Here all renormalization constants need to be evaluated at $\mathcal{O}(\alpha)$, i.e. from diagrams with a photon loop. Mass and field renormalization constants for the neutral particles $\chi^{0}, \tilde{\nu}_{\mu}$ as well as $\delta e^{(\gamma)}$ and $\delta Z_{A}^{(\gamma)}$ would be zero and do not have to be included. Field renormalization constants $\delta Z_{\chi, \tilde{\mu}}^{(\gamma)}$ could be introduced in order to cancel UV divergences of individual two-loop diagrams. Since they drop out in the end, we ignore them here.

Again, $\delta Z_{\mu}^{(\gamma)}$ has to be defined in the on-shell scheme. With this choice the photonic (i.e. $\mathcal{O}(\alpha)$ ) contribution to the muon field renormalization constant is UV and IR divergent, and it can be decomposed as

$$
\delta Z_{\mu}^{(\gamma)}=\delta Z_{\mu}^{(\gamma), \mathrm{UV}}+\delta Z_{\mu}^{(\gamma), \mathrm{IR}}=\frac{\alpha}{4 \pi}\left[-\frac{1}{\epsilon_{\mathrm{UV}}}-\frac{2}{\epsilon_{\mathrm{IR}}}-4+3 \mathrm{~L}\left(m_{\mu}^{2}\right)\right]
$$


The typical transcendental constants $\gamma_{E}$ and $\log 4 \pi$, which cancel in the end, are ignored throughout, and $\mathrm{L}\left(\mathrm{m}^{2}\right)$ has been defined in Eq. (12).

The choice of the renormalization scheme for the other parameters is more delicate. As discussed in Sec. 2 the photonic corrections appear as a subset of a larger class of two-loop contributions to $a_{\mu}$ in models such as the MSSM. In this context, the renormalization constants (23) are contributions to the full renormalization constants, $\delta m_{\chi^{ \pm}}^{\text {full }}=\delta m_{\chi^{ \pm}}^{(\gamma)}+\delta m_{\chi^{ \pm}}^{\text {remainder }}$ etc. This split is not unique, and by choosing a scheme for the constants (23) we effectively define precisely what we mean by "photonic corrections".

A natural choice for the mass counterterms are the on-shell values of the photonic contributions to the self energies, so we choose $\delta m_{\chi^{ \pm}}^{(\gamma)}=\Sigma_{\chi^{ \pm}}^{(\gamma)}\left(\not p=m_{\chi^{ \pm}}\right)$ and $\delta m_{\tilde{\mu}}^{2}(\gamma)=\Sigma_{\tilde{\mu}}^{(\gamma)}\left(p^{2}=m_{\tilde{\mu}}^{2}\right)$. The couplings $c, n$ appear only via the combinations $\mathcal{A}^{C, N}$ and $m_{\mu} \mathcal{B}^{C, N}$, see Eq. (9). Their renormalization transformations $c \rightarrow c+$ $\delta c^{(\gamma)}$ and $n \rightarrow n+\delta n^{(\gamma)}$ can be equivalently written as

$$
\begin{aligned}
\mathcal{A}^{C, N} & \rightarrow \mathcal{A}^{C, N}+\delta \mathcal{A}^{C, N(\gamma)}, \\
m_{\mu} \mathcal{B}^{C, N} & \rightarrow m_{\mu} \mathcal{B}^{C, N}+m_{\mu} \delta \mathcal{B}^{C, N(\gamma)}+\mathcal{B}^{C, N} \delta m_{\mu}^{(\gamma)} .
\end{aligned}
$$

In the class of models discussed in Sec. 2, the combinations $\mathcal{A}^{C, N}, \mathcal{B}^{C, N}$ depend only on details of the underlying theory, while the muon mass is a low-energy quantity. Hence a natural choice is to renormalize $\mathcal{A}^{C, N}$ and $\mathcal{B}^{C, N}$ in the $\overline{\mathrm{MS}}$ scheme and the muon mass in the on-shell scheme. Implicitly, this choice defines a renormalization scheme for the couplings $c, n$. We call the scheme defined in this way the "on-shell muon mass scheme" 5

As the major advantage, in this on-shell muon mass scheme the counterterm $\delta\left(c^{L} c^{R}\right)^{(\gamma)} /\left(c^{L} c^{R}\right)$ is set equal to $\delta m_{\mu}^{(\gamma)} / m_{\mu}+\delta \mathcal{B}^{C(\gamma)} / \mathcal{B}^{C}$ and similarly for $n^{L} n^{R}$, where the first term contains a large QED logarithm. This is important because in the considered class of models, $c^{L} c^{R}$ is proportional to the muon mass, and the full renormalization constants will satisfy $\delta\left(c^{L} c^{R}\right)^{\text {full }} /\left(c^{L} c^{R}\right)=\delta m_{\mu}^{(\gamma)} / m_{\mu}+\ldots$ and thus contain the same large QED logarithm.

Rewriting the renormalization transformations in terms of differential operators, we find a compact expression for the SUSY counterterm contributions in

\footnotetext{
${ }^{5}$ In other words, in the on-shell muon mass scheme, the $\overline{\mathrm{MS}}$-conditions on $\mathcal{A}^{C, N}$ and $\mathcal{B}^{C, N}$ imply that $\delta\left(\left|c^{L}\right|^{2}+\left|c^{R}\right|^{2}\right)^{(\gamma)}$ is a pure $\overline{\mathrm{MS}}$-quantity while $\delta\left(c^{L} c^{R}\right)^{(\gamma)}$ is not (and similarly for $c \rightarrow n)$. Note that this is consistent at the considered order in $m_{\mu}$, neglecting terms of $\mathcal{O}\left(m_{\mu}^{2}\right)$, but it makes difficult to individually solve for $\delta c^{L}$ and $\delta c^{R}$ as long as it is not specified which factor among $c^{L}, c^{R}$ is proportional to $m_{\mu}$.
} 
our on-shell muon mass scheme,

$$
\begin{aligned}
a_{\mu}^{\mathrm{ct}, \mathrm{SUSY}} & =\delta Z_{\mu}^{(\gamma)}\left(a_{\mu}^{\chi^{ \pm}}+a_{\mu}^{\chi^{0}}\right) \\
& +\left[\delta m_{\chi^{ \pm}}^{(\gamma)} \partial_{m_{\chi^{ \pm}}}+\sum_{i=L, R} \delta c^{i(\gamma, \overline{M S})} \partial_{c^{i}}+\frac{\delta m_{\mu}^{(\gamma, \mathrm{fin})}}{m_{\mu}} \mathcal{B}^{C} \partial_{\mathcal{B}^{C}}\right] a_{\mu}^{\chi^{ \pm}} \\
& +\left[\delta m_{\tilde{\mu}}^{2(\gamma)} \partial_{m_{\tilde{\mu}}^{2}}+\sum_{i=L, R} \delta n^{i(\gamma, \overline{M S})} \partial_{n^{i}}+\frac{\delta m_{\mu}^{(\gamma, \mathrm{fin})}}{m_{\mu}} \mathcal{B}^{N} \partial_{\mathcal{B}^{N}}\right] a_{\mu}^{\chi^{0}},
\end{aligned}
$$

where it is understood that the partial derivatives $\partial_{c^{i}}, \partial_{n^{i}}$ act on the couplings within $\mathcal{A}^{C, N}$ and $\mathcal{B}^{C, N}$. In spite of not using the $\overline{\mathrm{MS}}$-scheme for $c, n$ we find it convenient to express the counterterms in terms of the would-be $\overline{\mathrm{MS}}$-renormalization constants. For convenience, we provide the explicit values for the renormalization constants both in DREG and in DRED6

$$
\begin{aligned}
\frac{\delta m_{\chi^{ \pm}}^{(\gamma)}}{m_{\chi^{ \pm}}} & =\frac{\alpha}{4 \pi}\left[-\frac{3}{\epsilon}+3 \mathrm{~L}\left(m_{\chi^{ \pm}}^{2}\right)-4-\theta_{\mathrm{DRED}}\right], \\
\frac{\delta m_{\tilde{\mu}}^{2}(\gamma)}{m_{\tilde{\mu}}^{2}} & =\frac{\alpha}{4 \pi}\left[-\frac{3}{\epsilon}+3 \mathrm{~L}\left(m_{\tilde{\mu}}^{2}\right)-7\right] \\
\frac{\delta n^{L, R(\gamma, \overline{M S})}}{n^{L, R}} & =\frac{\alpha}{4 \pi}\left[-\frac{3}{2 \epsilon}+\frac{1}{2} \theta_{\mathrm{DRED}}\right], \\
\frac{\delta c^{L, R(\gamma, \overline{M S})}}{c^{L, R}} & =\frac{\alpha}{4 \pi}\left[-\frac{3}{\epsilon}-\theta_{\mathrm{DRED}}\right], \\
\frac{\delta m_{\mu}^{(\gamma, \mathrm{fin})}}{m_{\mu}} & =\frac{\alpha}{4 \pi}\left[3 \mathrm{~L}\left(m_{\mu}^{2}\right)-4\right] .
\end{aligned}
$$

The quantity $\theta_{\text {DRED }}=0$ in DREG and $\theta_{\text {DRED }}=1$ in the case of DRED.

We have checked that with these definitions all UV divergences cancel in the sum of the two-loop diagrams of Figs. 2a, 3a and the QED counterterm diagrams with the appropriate counterterm insertions of $\mathcal{O}\left(n^{2}, c^{2}\right)$. Likewise, all UV divergences cancel in the sum of the two-loop diagrams of Figs. 2b, 3b and the counterterms $a_{\mu}^{\text {ct,SUSY }}$. Furthermore, we have checked that the UV divergences cancel for each diagram (plus corresponding counterterm diagram) separately, if $\chi^{ \pm}$- and $\tilde{\mu}$-field renormalization is taken into account.

\footnotetext{
${ }^{6}$ See [46,47] for a more general analysis of the transition from DREG to DRED.
} 
The IR divergences cancel only in the following combinations:
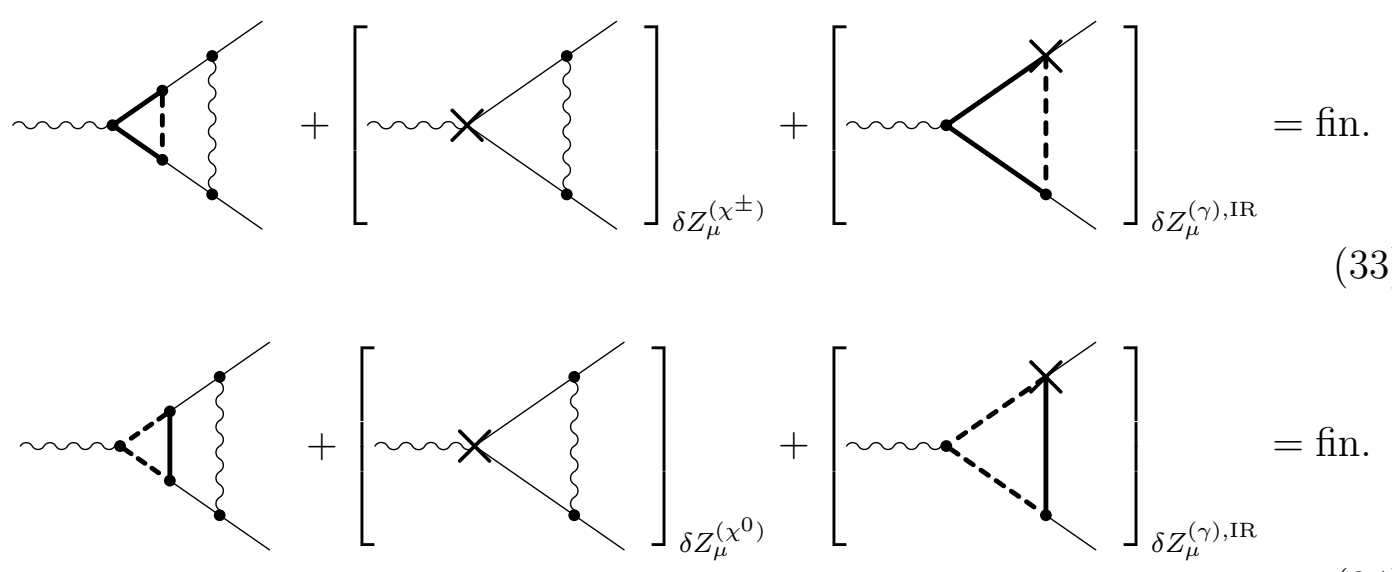

The unsuppressed terms of order $\left(m_{\mu} / M_{\mathrm{SUSY}}\right)^{0}$ cancel between the two-loop diagrams of Figs. 2, 3 and the QED counterterm diagrams.

Hence, after renormalization we obtain a UV and IR finite result of the order $\left(m_{\mu} / M_{\mathrm{SUSY}}\right)^{2}$ for the photonic two-loop contributions to $a_{\mu}$.

\section{Results}

The full result for the photonic two-loop corrections to $a_{\mu}$, defined by the sum of the two-loop diagrams of Sec. 3, $a_{\mu}^{2 \mathrm{~L} \text { diag }(\gamma)}$, and the counterterm contributions $a_{\mu}^{\mathrm{ct}, \mathrm{QED}}+a_{\mu}^{\mathrm{ct}, \mathrm{SUSY}}$, can be cast in a quite compact analytical form:

$$
\begin{aligned}
& a_{\mu}^{\chi^{ \pm}(\gamma)}=\frac{1}{16 \pi^{2}} \frac{\alpha}{4 \pi} \frac{m_{\mu}^{2}}{m_{\tilde{\nu}}^{2}}\left[\left(\frac{1}{12} \mathcal{A}^{C} F_{1}^{C}(x)+\frac{2 m_{\chi^{ \pm}}}{3} \mathcal{B}^{C} F_{2}^{C}(x)\right) 16 \log \frac{m_{\mu}}{m_{\tilde{\nu}}}\right. \\
& -\left(\frac{47}{72} \mathcal{A}^{C} F_{3}^{C}(x)+\frac{122 m_{\chi^{ \pm}}}{9} \mathcal{B}^{C} F_{4}^{C}(x)\right) \\
& \left.-\left(\frac{1}{2} \mathcal{A}^{C} F_{1}^{C}(x)+2 m_{\chi^{ \pm}} \mathcal{B}^{C} F_{2}^{C}(x)\right) \mathrm{L}\left(m_{\tilde{\nu}}^{2}\right)\right], \\
& a_{\mu}^{\chi^{0}(\gamma)}=\frac{1}{16 \pi^{2}} \frac{\alpha}{4 \pi} \frac{m_{\mu}^{2}}{m_{\tilde{\mu}}^{2}}\left[\left(-\frac{1}{12} \mathcal{A}^{N} F_{1}^{N}(x)+\frac{m_{\chi^{0}}}{3} \mathcal{B}^{N} F_{2}^{N}(x)\right) 16 \log \frac{m_{\mu}}{m_{\tilde{\mu}}}\right. \\
& -\left(-\frac{35}{72} \mathcal{A}^{N} F_{3}^{N}(x)+\frac{16 m_{\chi^{0}}}{9} \mathcal{B}^{N} F_{4}^{N}(x)\right) \\
& \left.+\left(\frac{1}{4} \mathcal{A}^{N} F_{1}^{N}(x)\right) \mathrm{L}\left(m_{\tilde{\mu}}^{2}\right)\right]
\end{aligned}
$$


where the kinematic variables are again defined as $x=m_{\chi^{ \pm}}^{2} / m_{\tilde{\nu}_{\mu}}^{2}$, or $x=m_{\chi^{0}}^{2} / m_{\tilde{\mu}}^{2}$, respectively. The functions $F_{3,4}^{C, N}$ are defined as

$$
\begin{aligned}
& F_{3}^{C}(x)=\frac{4}{141(1-x)^{4}}\left[(1-x)\left(151 x^{2}-335 x+592\right)\right. \\
& +6\left(21 x^{3}-108 x^{2}-93 x+50\right) \log x \\
& -54 x\left(x^{2}-2 x-2\right) \log ^{2} x \\
& \left.-108 x\left(x^{2}-2 x+12\right) \operatorname{Li}_{2}(1-x)\right] \text {, } \\
& F_{4}^{C}(x)=\frac{-9}{122(1-x)^{3}}\left[8\left(x^{2}-3 x+2\right)+\left(11 x^{2}-40 x+5\right) \log x\right. \\
& -2\left(x^{2}-2 x-2\right) \log ^{2} x \\
& \left.-4\left(x^{2}-2 x+9\right) \operatorname{Li}_{2}(1-x)\right] \text {, } \\
& F_{3}^{N}(x)=\frac{4}{105(1-x)^{4}}\left[(1-x)\left(-97 x^{2}-529 x+2\right)+6 x^{2}(13 x+81) \log x\right. \\
& \left.+108 x(7 x+4) \operatorname{Li}_{2}(1-x)\right], \\
& F_{4}^{N}(x)=\frac{-9}{4(1-x)^{3}}\left[(x+3)(x \log x+x-1)+(6 x+2) \operatorname{Li}_{2}(1-x)\right],
\end{aligned}
$$

so that they are normalized to unity for $x=1$. The logarithms in the first lines of Eqs. (35), (36) reproduce the result of Ref. [36] for the leading QED-logarithms, $\frac{4 \alpha}{\pi} \log \frac{m_{\mu}}{M_{\mathrm{SUSY}}}$ times the one-loop result. As can be easily seen, these logarithms are negative and reduce the one-loop result by (7 . .9)\% for SUSY masses between $100 \ldots 1000 \mathrm{GeV}$. It is interesting to note that the remaining contributions are typically also negative and lead to a further reduction. In particular, for $x=1$ the $F_{4}^{C}$-term alone leads to an additional $1.2 \%$ reduction of the corresponding one-loop contribution.

For our further discussion of the result we specialize to the case of the MSSM. There, the one-loop and the photonic two-loop contributions are given by

$$
\begin{aligned}
a_{\mu}^{\mathrm{SUSY}, 1 \mathrm{~L}} & =\sum_{k} a_{\mu}^{\chi^{ \pm}}+\sum_{i, m} a_{\mu}^{\chi^{0}}, \\
a_{\mu}^{\mathrm{SUSY}, 2 \mathrm{~L}(\gamma)} & =\sum_{k} a_{\mu}^{\chi^{ \pm}(\gamma)}+\sum_{i, m} a_{\mu}^{\chi^{0}(\gamma)},
\end{aligned}
$$

where the SUSY masses and couplings have to be inserted appropriately, as discussed in Sec. 2.

As a general remark, the photonic two-loop corrections depend on the same MSSM parameters as the one-loop contributions, and owing to the structure of the analytical results we can expect the overall parameter dependence to be similar. In particular, the photonic corrections are proportional to $\tan \beta$, just like 


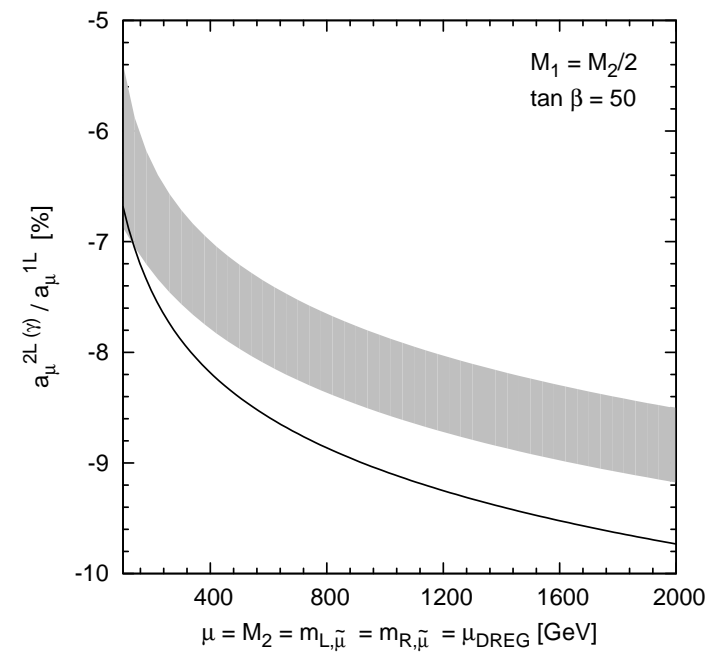

(a)

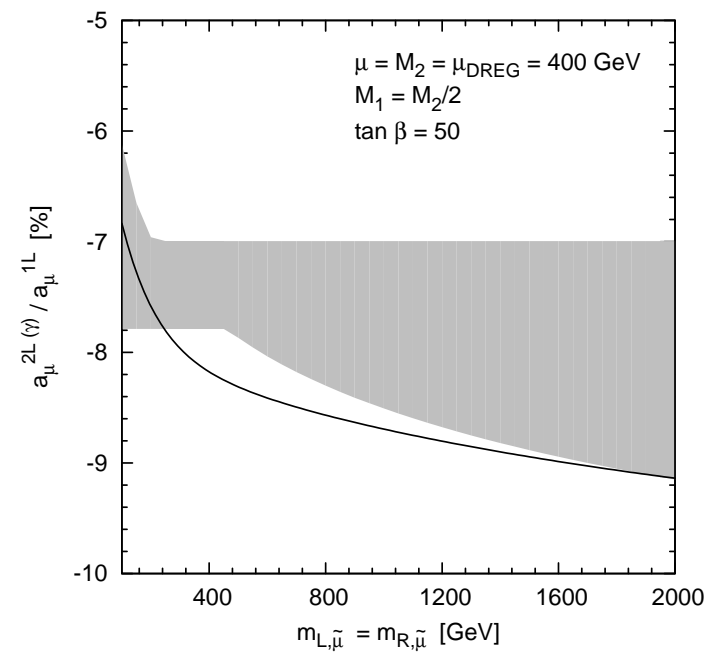

(b)

Figure 6: (a) Photonic two-loop corrections, relative to the MSSM one-loop contributions, as a function of a common SUSY mass scale, see Eq. (43). (b) The same, as a function of $m_{L, \tilde{\mu}}=m_{R, \tilde{\mu}}$ with fixed $M_{2}=\mu=\mu_{\mathrm{DREG}}=400 \mathrm{GeV}$. Our result is shown as a continuous line, the leading-log approximation as a grey band.

the one-loop contributions. Hence, in all our plots we choose a fixed large value $\tan \beta=50$ and plot the ratio of the two- and one-loop contributions.

Figure 6a shows the numerical impact of the the photonic corrections, relative to the one-loop result, for a simple, generic case 7 We choose the fundamental SUSY mass parameters for the Higgsino, wino, left- and right-handed smuon equal,

$$
\mu=M_{2}=m_{L, \tilde{\mu}}=m_{R, \tilde{\mu}}=\mu_{\mathrm{DREG}}
$$

only the bino mass parameter is determined by the GUT relation $M_{1}=M_{2} / 2$, and $\tan \beta=50$. The exact result for the ratio of the photonic corrections to the one-loop result is denoted by the continuous line. For comparison we also show the leading-log result, which, without further knowledge, can only be computed with $M_{\mathrm{SUSY}}$ in a reasonable range. We choose $M_{\mathrm{SUSY}}$ in the range between the minimum and maximum of the mass eigenvalues $m_{\chi^{ \pm, 0}}, m_{\tilde{\mu}, \tilde{\nu} \mu}$ and represent the result by the grey band.

Fig. 6b shows a similar plot where $M_{2}=\mu=\mu_{\mathrm{DREG}}=400 \mathrm{GeV}$ fixed and $m_{L, \tilde{\mu}}=m_{R, \tilde{\mu}}$ are varied.

\footnotetext{
${ }^{7}$ In the numerical analysis we follow Refs. 33, 34, 37 and parametrize the one-loop result in terms of the muon decay constant $G_{\mu}$, i.e. within $a_{\mu}^{\mathrm{SUSY}, 1 \mathrm{~L}}$ we replace $\pi \alpha / s_{W}^{2} \rightarrow \sqrt{2} G_{\mu} M_{W}^{2}$, in order to absorb further universal two-loop corrections. This does not influence the explicit factor $\alpha$ within $a_{\mu}^{\mathrm{SUSY}, 2 \mathrm{~L}(\gamma)}$, which is defined in the on-shell scheme.
} 


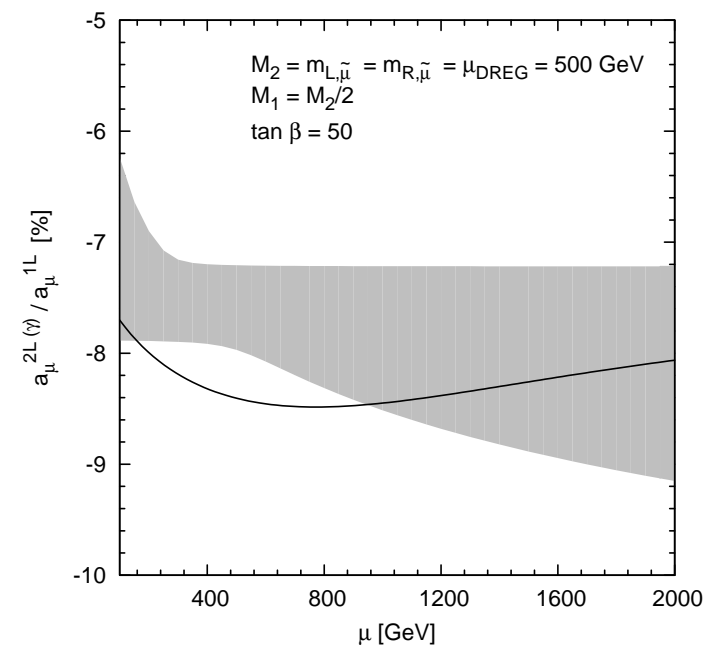

(a)

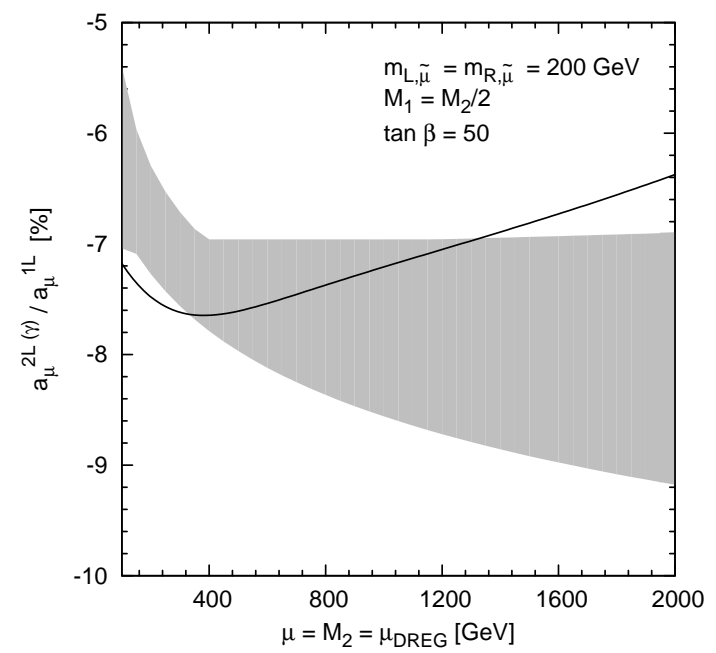

(b)

Figure 7: (a) Photonic two-loop corrections, relative to the MSSM one-loop contributions, as a function of $\mu$. (b) Photonic two-loop corrections, relative to the MSSM one-loop contributions, as a function of $\mu=M_{2}$. The other SUSY parameters are fixed as shown in the figure.

Not surprisingly, owing to the additional negative non-logarithmic contributions the exact result lies outside the band for the leading-log estimate. The leading-log result approximates the exact result best if we choose $M_{\text {SUSY }}$ as the maximum of all SUSY masses (because of the negative sign, this corresponds to the lower border of the band).

In order to understand the behaviour of the MSSM result in more detail it is important to note that the MSSM contributions are enhanced by $\tan \beta$, but this enhancement only affects the terms involving $\mathcal{B}^{C, N}$; hence the $\mathcal{A}^{C, N_{-}}$ terms are comparatively unimportant. The $\tan \beta$-enhanced terms can be well approximated by mass-insertion diagrams [20, 32], with propagating gauginos and Higgsinos and insertions of the off-diagonal entries of the chargino/neutralino mass matrices. This implies that in the sums over mass eigenstates in (41), (42), intricate cancellations take place. The one-loop result depends sensitively on mass differences and is mainly determined by the derivatives of the $F_{i}^{j}[20$.

The same discussion can be carried out for the photonic two-loop corrections. For the scenario with equal SUSY mass parameters the dominant terms are the ones involving $F_{2}^{C}$ and $F_{4}^{C}$, where $F_{2}^{C \prime}(1)=-3 / 4$ and $F_{4}^{C \prime}(1)=-45 / 122$. The smaller derivative of $F_{4}^{C}$ partially compensates the large coefficient in (35), and for this reason the exact result lies only slightly below the leading-log estimate for large $M_{\mathrm{SUSY}}$.

Fig. 7a analyses the dependence on the Higgsino mass parameter $\mu$, keeping all other SUSY mass parameters fixed. For large or small $\mu / M_{2}$ there is a large spread in the chargino mass spectrum, and the uncertainty of the leading-log 

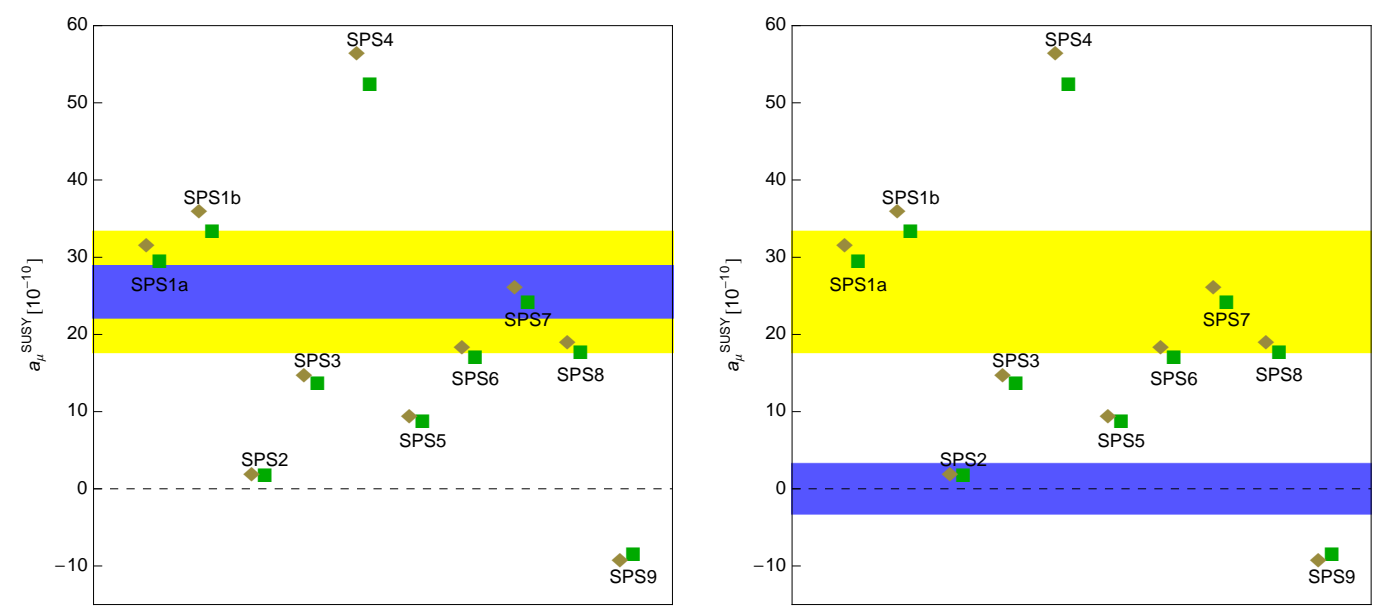

Figure 8: The predictions for $a_{\mu}^{\text {SUSY }}$ for the Snowmass Points and Slopes benchmark scenarios [48]. The wide band corresponds to the present $1 \sigma$ region of Eq. (3). The narrow band represents the foreseen (SM theory-limited) improved precision if the new $g-2$ measurement is carried out [29], given the same central value (left) or assuming the deviation vanishes (right). The square points denote the full result for $a_{\mu}^{\mathrm{SUSY}}$ including all known two-loop corrections, the diamond points are computed without the photonic corrections.

estimate is large. As the figure shows, in these parameter regions the exact result lies in the leading-log band.

The mass-insertion diagrams also show that the $\mu$-dependence is non-trivial. For small $\mu$, the chargino diagrams dominate. For large $\mu$, the diagram with bino exchange and left-right smuon transition can dominate [20] - it is the unique diagram that increases linearly with $\mu$. But this behaviour is approximately the same at the one- and two-loop level, and therefore the ratio shown in the figure is almost constant. Nevertheless, for large $\mu$ positive photonic contributions start to partially cancel the leading logarithms.

Fig. $7 \mathrm{~b}$ shows the behaviour if all chargino and neutralino masses are varied together, $\mu=M_{2}$ and $M_{1}=M_{2} / 2$, with fixed smuon/sneutrino mass parameters. Similarly to Fig. 7a, positive two-loop contributions can become important at large $\mu$, and the exact result can even lie above the leading-log band.

Finally we consider the results for the SPS benchmark points [48]. E.g. SPS1a and SPS1b lead to $a_{\mu}^{\text {SUSY }}$ close to the observed deviation (3). SPS1a has $\tan \beta=$ 10 and quite small SUSY masses, SPS1b has $\tan \beta=30$ and slightly larger SUSY 
masses. The results for these points are, in units of $10^{-10}$,

\begin{tabular}{|c|c|c|c|}
\hline & $a_{\mu}^{\text {SUSY,1L }}$ & $a_{\mu}^{\text {SUSY,2L leading log }}$ & $a_{\mu}^{\text {SUSY,2L }(\gamma)}$ \\
\hline SPS1a & 30.49 & $-1.93 \ldots-2.32$ & -2.18 \\
\hline SPS1b & 33.34 & $-2.27 \ldots-2.63$ & -2.59 \\
\hline
\end{tabular}

Fig. 8 shows a graphical distribution of the ten SPS benchmark predictions for $a_{\mu}^{\mathrm{SUSY}}$, computed with all known one- and two-loop corrections, and compared with the experimental and SM value. The discriminating power of the current and an improved $a_{\mu}$ determination is evident. The plot also displays the results obtained neglecting the photonic corrections. For several benchmark points, the difference is as large as one sigma of the future $\Delta a_{\mu}(\exp -\mathrm{SM})$.

\section{Matching the result to a full MSSM calcula- tion}

Our results have been presented in such a way that they can be easily utilized as building blocks in a full calculation of all two-loop corrections to $a_{\mu}^{\mathrm{SUSY}, 1 \mathrm{~L}}$ in the MSSM. This is useful because two technical problems are concentrated in the photonic corrections: the appearance of IR divergences and of terms which are not suppressed by powers of $m_{\mu}$ divided by a heavy mass of the order of the weak scale or SUSY scale.

As discussed in Secs. 1, 2, the SUSY two-loop corrections to SM one-loop diagrams are already known. What remains to be computed are the two-loop corrections to SUSY one-loop diagrams, denoted by $a_{\mu}^{\mathrm{SUSY}, 2 \mathrm{~L}(\mathrm{~b})}$ in Ref. [20]. These contributions are defined as the two-loop diagrams where the $\mu$-lepton number is carried by a $\tilde{\mu}$ and/or $\tilde{\nu}_{\mu}$ line, plus corresponding counterterm diagrams.

Similar to our photonic corrections, these full contributions can be decom-

posed as $a_{\mu}^{2 \mathrm{~L} \text { diag full }}+a_{\mu}^{\text {ct,QED full }}+a_{\mu}^{\text {ct,SUSY full }}+a_{\mu}^{\text {ct,rem }}$. Here $a_{\mu}^{2 \mathrm{~L} \text { diag full }}$ denote the genuine two-loop diagrams and the counterterm contributions have been split into the QED counterterm diagrams of the form in Fig. 4, the SUSY counterterm diagrams of the form in Fig. 5, and all other remaining counterterm diagrams. Hence we can write

$$
\begin{aligned}
a_{\mu}^{\mathrm{SUSY}, 2 \mathrm{~L}(\mathrm{~b})} & =a_{\mu}^{\mathrm{SUSY}, 2 \mathrm{~L}(\gamma)} \\
& +\left(a_{\mu}^{2 \mathrm{~L} \operatorname{diag} \text { full }}-\sum a_{\mu}^{2 \mathrm{~L} \operatorname{diag}(\gamma)}\right) \\
& +\left(a_{\mu}^{\mathrm{ct}, \mathrm{QED} \text { full }}-\sum a_{\mu}^{\mathrm{ct}, \mathrm{QED}}\right) \\
& +\left(a_{\mu}^{\mathrm{ct}, \mathrm{SUSY} \text { full }}-\sum a_{\mu}^{\mathrm{ct}, \mathrm{SUSY}}\right) \\
& +\left(a_{\mu}^{\mathrm{ct}, \mathrm{rem}}\right)
\end{aligned}
$$


where the appropriate summation over the chargino, neutralino and smuon indices of each term is implied.

Setting up the full calculation in this way has several technical advantages.

- In the difference of the genuine loop diagrams (2nd line of Eq. (44)), simply all the photonic diagrams of Figs. 2, 3 drop out. In other words, only the non-photonic two-loop diagrams need to be evaluated. As an advantage, these are all individually infrared finite and suppressed by $m_{\mu}^{2}$ divided by a heavy mass squared.

- The difference of the QED counterterm diagrams (3rd line of Eq. (44)) vanishes. The reason is that the appearing QED counterterm insertions and renormalization constants, see Eq. (22), have to be defined in the same way, in the on-shell scheme, in the full theory and for the photonic corrections. Therefore, the QED counterterm diagrams, which are again partially infrared divergent, need not be re-evaluated in the full calculation. All counterterm diagrams that remain to be calculated are individually infrared finite and suppressed by $m_{\mu}^{2}$ divided by a heavy mass squared.

The only subtlety arises in the 4th line of Eq. (44), in connection with the SUSY counterterm diagrams of Fig. 5. The renormalization constants (23) appearing within these diagrams are different in the full MSSM and in our calculation. In the full theory, $\delta Z_{\mu}$ must also be defined in the on-shell scheme, but further diagrams contribute to it. Mass and field renormalization constants for the neutral particles do not vanish any more. Finally, for the SUSY masses and couplings not even the same renormalization scheme can be used in the full theory 8

Nevertheless, it is possible to choose any desired MSSM renormalization

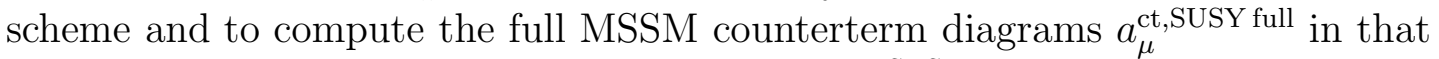
scheme. Then simply our counterterm result $a_{\mu}^{\text {ct,SUSY }}$, which has been given in Eq. (27), has to be explicitly subtracted. The choice of the regularization scheme DREG or DRED for the counterterms has to match the choice for the two-loop diagrams. In this way, the full result corresponding to the desired MSSM renor-

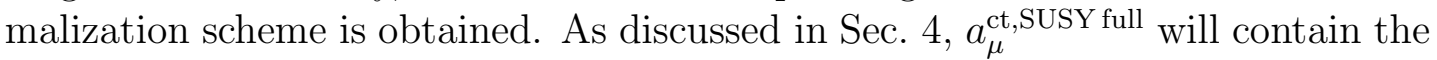
same large QED logarithm arising from $\delta m_{\mu}$ within the SUSY coupling renormalization that is already contained in $a_{\mu}^{\mathrm{ct}, \mathrm{SUSY}}$. Therefore, the difference in the 4th line of Eq. (44) is free of large QED logarithms.

\footnotetext{
${ }^{8}$ In the MSSM, supersymmetry together with $\mathrm{SU}(2) \times \mathrm{U}(1)$ gauge invariance implies correlations between the SUSY masses and the different couplings $c, n$, which must be reflected in the renormalization scheme, see e.g. 49 51. But they cannot be reflected in the purely photonic corrections since these are not invariant under the full symmetry of the MSSM.
} 


\section{Conclusions}

In the present paper the photonic two-loop corrections to the muon magnetic moment $a_{\mu}$ in the MSSM and a wider class of models have been evaluated exactly. The photonic corrections are defined as the two-loop diagrams which contain a photon loop attached to a SUSY one-loop diagram, plus the corresponding counterterm diagrams. The counterterms are defined in the "on-shell muon mass scheme", which is natural for the models considered here and in Ref. [36] - all QED quantities and all masses are renormalized in the on-shell scheme, but the purely high-scale parameters are $\overline{\mathrm{MS}}$-renormalized.

Our result reproduces the large logarithms of Ref. [36] and provides the exact result for the additional subleading logarithms, dilogarithms and non-logarithmic terms. The leading logarithm has an intrinsic uncertainty because it could be evaluated with a small, large, or intermediate SUSY mass. For the typical SUSY scenarios considered here, the leading logarithm amounts to around $-7 \%$ of $a_{\mu}^{\mathrm{SUSY}, 1 \mathrm{~L}}$ if the smallest SUSY mass is used; the additional terms are in the range $(0.5 \ldots-2) \%$ and thus typically lead to a further reduction of $a_{\mu}^{\mathrm{SUSY}, 1 \mathrm{~L}}$. However, for large $\mu$, the additional terms can have a positive sign and can partially compensate the leading logarithm.

It is interesting to compare the photonic two-loop corrections to other known SUSY two-loop contributions to the muon magnetic moment. Another universal two-loop correction is the $(\tan \beta)^{2}$-correction arising from a shift of the muon Yukawa coupling [37. In a large part of the MSSM parameter space, particularly for approximately degenerate SUSY masses, the photonic and the $(\tan \beta)^{2}$ corrections are the largest two-loop effects. While the photonic corrections are negative, the $(\tan \beta)^{2}$-corrections are positive (for positive $a_{\mu}^{\mathrm{SUSY}, 1 \mathrm{~L}}$ ) and can overcompensate the photonic corrections for large $\tan \beta$. The SUSY two-loop corrections to Standard Model one-loop diagrams [33, 34] amount to around $2 \%$ of $a_{\mu}^{\mathrm{SUSY}, 1 \mathrm{~L}}$ for degenerate masses, but in special cases with large mass splittings or in cases where the one-loop contributions are suppressed, they can become dominant.

Importantly, with the exact two-loop computation the theory error arising from unknown photonic corrections has been reduced. The remaining theory error due to unknown photonic three-loop corrections can be estimated by comparing with the electroweak contributions in the Standard Model. There, the photonic three-loop corrections amount to only $1 \%$ of the photonic two-loop corrections. Based on that, we can estimate the unknown photonic three-loop corrections to $a_{\mu}^{\mathrm{SUSY}, 1 \mathrm{~L}}$ to be less than $\mathcal{O}\left(0.1 \times 10^{-10}\right)$ and thus negligible.

Nevertheless, in order to make full use of the expected and intended improve-

ments of $a_{\mu}^{\exp }$ and $a_{\mu}^{\mathrm{SM}}$ for SUSY phenomenology, the SUSY theory error should be further reduced. Our calculation can also help in computing the remaining SUSY two-loop contributions to $a_{\mu}$. If the full calculation is organized as in Eq. (44), only non-photonic diagrams and non-QED counterterm diagrams remain to 
be computed. These are all infrared finite and suppressed by the required $m_{\mu}^{2}$ factor and contain no further large QED-logarithm.

\section{Acknowledgments}

This work was supported by the German Research Foundation DFG through Grant No. STO876/1-1.

\section{References}

[1] G.W. Bennett, et al., (Muon $(g-2)$ Collaboration), Phys. Rev. D 73, 072003 (2006).

[2] B. L. Roberts, arXiv:1001.2898 [hep-ex].

[3] M. N. Achasov et al. [SND Collaboration], J. Exp. Theor. Phys. 103 (2006) 380 [Zh. Eksp. Teor. Fiz. 130 (2006) 437].

[4] R. R. Akhmetshin et al. [CMD-2 Collaboration], Phys. Lett. B 648 (2007) 28.

[5] A. Aloisio et al. [KLOE Collaboration], Phys. Lett. B 606 (2005) 12;

F. Ambrosino et al., [KLOE Collaboration], Phys. Lett. B 670 (2009) 285,

S. E. Muller, arXiv:0912.2205 [hep-ex].

[6] B. Aubert et al. [BaBar Collaboration], Phys. Rev. D 70, 072004 (2004), Phys. Rev. D 71, 052001 (2005), Phys. Rev. D 73, 052003 (2006), Phys. Rev. Lett. 103 (2009) 231801.

[7] K. Hagiwara, A. D. Martin, D. Nomura and T. Teubner, Phys. Lett. B 649 (2007) 173 arXiv:hep-ph/0611102.

[8] T. Teubner, K. Hagiwara, R. Liao, A. D. Martin and D. Nomura, arXiv:1001.5401 [hep-ph].

[9] Eduardo de Rafael arXiv:0809.3085.

[10] F. Jegerlehner and A. Nyffeler, Phys. Rept. 477 (2009) 1.

[11] M. Davier et al., Eur. Phys. J. C 66, 127 (2010).

[12] M. Davier, A. Hoecker, B. Malaescu, C. Z. Yuan and Z. Zhang, Eur. Phys. J. C 66, 1 (2010).

[13] R. Alemany, M. Davier and A. Hocker, Eur. Phys. J. C 2 (1998) 123. 
[14] S. Ghozzi and F. Jegerlehner, Phys. Lett. B 583, 222 (2004).

[15] Kirill Melnikov, Arkady Vainshtein, Theory of the Muon Anomalous Magnetic Moment (Springer Tracts in Modern Physics).

[16] M. Benayoun, P. David, L. DelBuono, O. Leitner and H. B. O'Connell, Eur. Phys. J. C 55, 199 (2008); M. Benayoun, P. David, L. DelBuono and O. Leitner, arXiv:0907.5603 [hep-ph].

[17] J. Prades, E. de Rafael and A. Vainshtein, arXiv:0901.0306 [hep-ph].

[18] A. Nyffeler, Phys. Rev. D 79, 073012 (2009).

[19] A. Czarnecki and W. J. Marciano, Phys. Rev. D 64 (2001) 013014.

[20] D. Stöckinger, J. Phys. G 34 (2007) R45.

[21] S. P. Martin and J. D. Wells, Phys. Rev. D 67 (2003) 015002.

[22] R. R. de Austri, R. Trotta and L. Roszkowski, JHEP 0605 (2006) 002; F. Feroz, M. P. Hobson, L. Roszkowski, R. R. de Austri and R. Trotta, arXiv:0903.2487 [hep-ph].

[23] B. C. Allanach, K. Cranmer, C. G. Lester and A. M. Weber, JHEP 0708, 023 (2007); F. Feroz, B. C. Allanach, M. Hobson, S. S. AbdusSalam, R. Trotta and A. M. Weber, JHEP 0810 (2008) 064; S. S. AbdusSalam, B. C. Allanach, M. J. Dolan, F. Feroz and M. P. Hobson, arXiv:0906.0957 [hep-ph]; S. S. AbdusSalam, B. C. Allanach, F. Quevedo, F. Feroz and M. Hobson, arXiv:0904.2548 [hep-ph].

[24] J. R. Ellis, S. Heinemeyer, K. A. Olive, A. M. Weber and G. Weiglein, JHEP 0708 (2007) 083; O. Buchmueller et al., JHEP 0809 (2008) 117; O. Buchmueller et al., Eur. Phys. J. C 64, 391 (2009).

[25] P. Bechtle, K. Desch, M. Uhlenbrock and P. Wienemann, Eur. Phys. J. C 66, 215 (2010).

[26] D. W. Hertzog, J. P. Miller, E. de Rafael, B. Lee Roberts and D. Stöckinger, arXiv:0705.4617.

[27] R. Lafaye, T. Plehn, M. Rauch and D. Zerwas, Eur. Phys. J. C 54, 617 (2008).

[28] M. Alexander, S. Kreiss, R. Lafaye, T. Plehn, M. Rauch, and D. Zerwas, Chapter 9 in M. M. Nojiri et al., arXiv:0802.3672 [hep-ph]. 
[29] D. W. Hertzog, B. Lee Roberts et al., Fermilab Proposal P-989, March 2009, http://www.fnal.gov/directorate/program_planning/ Mar2009PACPublic/PACMarch09AgendaPublic.htm; B. L. Roberts, arXiv:1001.2898 [hep-ex].

[30] T. Mibe, Proceedings to PhiPsio9, Chin.Phys.C.

[31] J. L. Lopez, D. V. Nanopoulos and X. Wang, Phys. Rev. D 49, 366 (1994); U. Chattopadhyay and P. Nath, Phys. Rev. D 53, 1648 (1996);

[32] T. Moroi, Phys. Rev. D 53 (1996) 6565 [Erratum-ibid. 56 (1997) 4424].

[33] S. Heinemeyer, D. Stöckinger and G. Weiglein, Nucl. Phys. B 690 (2004) 62 .

[34] S. Heinemeyer, D. Stöckinger and G. Weiglein, Nucl. Phys. B 699 (2004) 103.

[35] T. F. Feng, L. Sun and X. Y. Yang, Phys. Rev. D 77 (2008) 116008; Nucl. Phys. B 800 (2008) 221; T. F. Feng and X. Y. Yang, Nucl. Phys. B 814 (2009) 101.

[36] G. Degrassi and G. F. Giudice, Phys. Rev. D 58 (1998) 053007.

[37] S. Marchetti, S. Mertens, U. Nierste and D. Stöckinger, Phys. Rev. D 79, 013010 (2009).

[38] T. F. Feng, X. Q. Li, L. Lin, J. Maalampi and H. S. Song, Phys. Rev. D 73, 116001 (2006).

[39] S. Martin and J. Wells, Phys. Rev. D 64 (2001) 035003.

[40] A. Signer and D. Stöckinger, Phys. Lett. B 626 (2005) 127; Nucl. Phys. B 808 (2009) 88.

[41] A. Czarnecki and B. Krause, Nucl. Phys. Proc. Suppl. 51C (1996) 148 [arXiv:hep-ph/9606393].

[42] J. Küblbeck, M. Böhm, and A. Denner, Comput. Phys. Commun. 60 (1990) 165 ;

T. Hahn, Comput. Phys. Commun. 140 (2001) 418.

[43] T. Hahn and C. Schappacher, Comput. Phys. Commun. 143 (2002) 54.

[44] G. Weiglein, R. Scharf and M. Böhm, Nucl. Phys. B 416 (1994) 606; G. Weiglein, R. Mertig, R. Scharf and M. Böhm, in New Computing Techniques in Physics Research 2, ed. D. Perret-Gallix (World Scientific, Singapore, 1992), p. 617. 
[45] V. A. Smirnov, Mod. Phys. Lett. A 10 (1995) 1485 arXiv:hep-th/9412063; Applied Asymptotic Expansions in Momenta and Masses, Springer Verlag, Berlin (2002).

[46] S. P. Martin and M. T. Vaughn, Phys. Lett. B 318 (1993) 331.

[47] L. Mihaila, Phys. Lett. B 681 (2009) 52.

[48] B. C. Allanach et al., in Proc. of the APS/DPF/DPB Summer Study on the Future of Particle Physics (Snowmass 2001) ed. N. Graf, Eur. Phys. J. C 25 (2002) 113.

[49] W. Hollik, E. Kraus, M. Roth, C. Rupp, K. Sibold and D. Stöckinger, Nucl. Phys. B 639 (2002) 3.

[50] T. Fritzsche and W. Hollik, Eur. Phys. J. C 24, 619 (2002).

[51] W. Hollik and H. Rzehak, Eur. Phys. J. C 32 (2003) 127. 